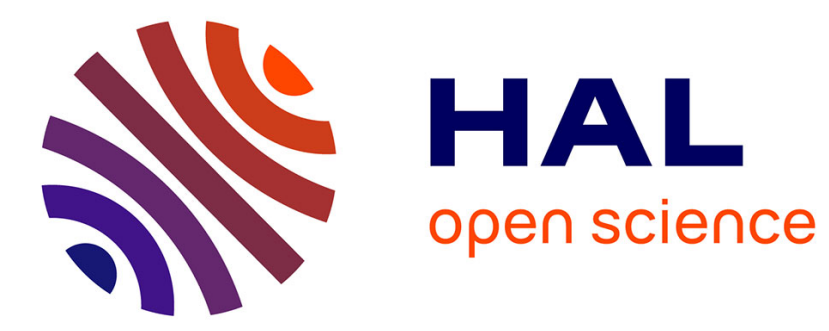

\title{
Deuxième partie. - Structure des métaux et alliages amorphes
}

\author{
J. Bletry
}

\section{To cite this version:}

J. Bletry. Deuxième partie. - Structure des métaux et alliages amorphes. Revue de Physique Appliquée, 1980, 15 (6), pp.1019-1031. 10.1051/rphysap:019800015060101901 . jpa-00244818

\section{HAL Id: jpa-00244818 https://hal.science/jpa-00244818}

Submitted on 1 Jan 1980

HAL is a multi-disciplinary open access archive for the deposit and dissemination of scientific research documents, whether they are published or not. The documents may come from teaching and research institutions in France or abroad, or from public or private research centers.
L'archive ouverte pluridisciplinaire HAL, est destinée au dépôt et à la diffusion de documents scientifiques de niveau recherche, publiés ou non, émanant des établissements d'enseignement et de recherche français ou étrangers, des laboratoires publics ou privés. 


\title{
Deuxième partie. - Structure des métaux et alliages amorphes
}

\author{
J. Bletry \\ Laboratoire de Thermodynamique et Physico-Chimie métallurgiques (L.A. 29), E.N.S.E.E.G., B.P. ${ }^{\circ}{ }^{44}$, \\ 38401 Saint Martin d'Hères, France
}

Introduction. - Les recherches sur les matériaux amorphes se développent avec rapidité. Cet essor est encouragé par les applications prometteuses entrevues dans des domaines divers tels que l'informatique (mémoires à bulles), l'énergie solaire (photopiles) ou la métallurgie (propriétés mécaniques, électriques et magnétiques, résistance à la corrosion).

Toutefois les études structurales, nécessaires à la compréhension des propriétés macroscopiques de ces matériaux, se heurtent à des difficultés expérimentales inhérentes aux états métastables en général et à l'état amorphe en particulier. En effet, la structure d'un alliage amorphe peut varier selon le mode de préparation, l'épaisseur, l'état de division... ou l'histoire thermique de l'échantillon examiné. Il faut donc contrôler tous ces facteurs pour définir sans ambiguïté l'état amorphe considéré. D'autre part, l'étude des structures amorphes à l'aide des techniques de diffraction exige un grand soin car des détails observés sur les diagrammes expérimentaux influent souvent de manière déterminante sur la description de l'ordre local.

Enfin, l'absence des familières périodicités cristallines explique que l'état amorphe constitue encore un sujet théorique riche et ouvert.

Nous nous limiterons ici à présenter les principales méthodes de diffraction et quelques modèles structuraux géométriques applicables aux métaux et alliages amorphes. En comparant les résultats expérimentaux et théoriques obtenus dans le cas particulier des alliages cobalt-phosphore, nous montrerons les possibilités, les limites et les perspectives de ce type d'étude.
1. Méthodes diffractométriques. -1.1 RAPPELS. - 1.1.1 Facteur de structure et fonction de corrélation de paire. - Dans une expérience typique de diffraction l'échantillon est bombardé par un faisceau de particules (électrons, rayons $\mathrm{X}$, neutrons...) de longueur d'onde $\lambda$. Un détecteur couvrant l'angle solide $\Delta \Omega$ permet de mesurer le nombre de particules diffusées par seconde sous l'angle $2 \theta$. Moyennant certaines corrections, on déduit de cette intensité la section efficace différentielle de diffusion cohérente (en $\mathrm{cm}^{2}$ par stéradian). Dans le cas d'un amorphe monoatomique, cette section efficace s'écrit :

$$
\frac{\mathrm{d} \sigma}{\mathrm{d} \Omega}=|f(Q)|^{2} S(Q)
$$

où $Q=4 \pi \sin \theta / \lambda$ désigne le module du vecteur de diffusion (élastique) $\mathbf{Q}$.

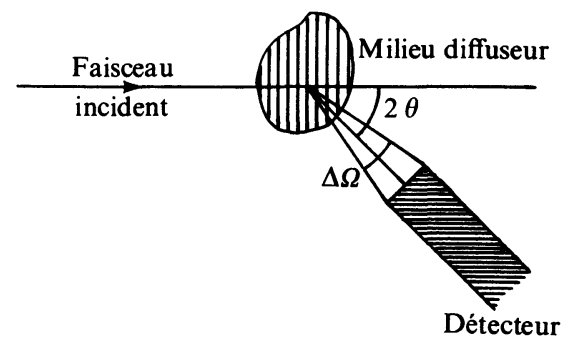

Connaissant la longueur de diffusion cohérente $f(Q)$ qui caractérise l'interaction rayonnement-diffuseur, on peut alors déterminer le facteur de structure du milieu diffuseur :

$$
S(Q)=1+\rho \int[P(r)-1] \mathrm{e}^{i \mathbf{Q r}} \mathrm{d}_{3} \mathbf{r}
$$


où $\rho=N / V$ représente le nombre de diffuseurs par unité de volume et $P(r)$ la fonction de corrélation (ou de distribution) de paire c'est-à-dire la probabilité par unité de volume de trouver un diffuseur à une distance $r$ d'un autre diffuseur (normalisée à 1 lorsque $r$ tend vers l'infini).

La relation inverse :

$$
\begin{aligned}
P(r)=1+\frac{1}{(2 \pi)^{3}} \frac{1}{\rho} \int_{0}^{\infty}[S(Q)-1] \times \\
\quad \times \frac{\sin (Q r)}{Q r} 4 \pi Q^{2} \mathrm{~d} Q
\end{aligned}
$$

permet de calculer la fonction $P(r)$ à partir des mesures de facteur de structure et de densité. Mais, cette transformation de Fourier est entachée d'une erreur de coupure plus ou moins importante car l'intervalle de $Q$ expérimental est fini. C'est pourquoi nous préférons présenter et comparer les résultats expérimentaux et théoriques obtenus dans l'espace $Q$ des facteurs de structure ( $(2$ et 3$)$.

1 1.2 Longueurs de diffusion. - 1.1.2.1 Rayons X - La longueur de diffusion introduite précédemment est proportionnelle à la transformée de Fourier du potentiel d'interaction diffuseur-rayonnement. Dans le cas des rayons $\mathrm{X}$ (non polarisés) $f_{\mathrm{x}}(Q)$ s'écrit :

$$
f_{\mathbf{x}}(Q)=r_{\mathrm{e}} \sqrt{\frac{1+\cos ^{2} 2 \theta}{2}} F_{\mathrm{e}}(Q)
$$

où $r_{\mathrm{e}}=2,82 \times 10^{-13} \mathrm{~cm}$ désigne le rayon classique de l'électron,

$F_{\mathrm{e}}(Q)=\int \rho_{\mathrm{e}}(r) \mathrm{e}^{i \mathbf{Q r}} \mathrm{d}_{3} \mathbf{r}$ le facteur de forme électronique

et $\rho_{\mathrm{e}}(r)$ la densité électronique totale de l'atome diffuseur.

La longueur de diffusion des rayons $\mathrm{X}$ est donc proportionnelle au numéro atomique $Z=F_{\mathrm{e}}(0)$ et décroît lorsque $Q$ augmente.

1.1.2.2 Neutrons. - La longueur de diffusion neutronique est la somme de deux termes qui correspondent respectivement aux interactions avec les noyaux et (éventuellement) avec les électrons magnétiques.

La portée des forces nucléaires étant très petite devant les longueurs d'onde cristallographiques et les distances interatomiques, la longueur de diffusion nucléaire $b$ est indépendante de $Q$. Par ailleurs, cette longueur de diffusion dépend du noyau considéré et de l'orientation de son spin. Un élément chimique est ainsi caractérisé par sa longueur de diffusion cohérente $b_{\text {coh }}$ qui est une moyenne sur tous les isotopes et les orientations des spins nucléaires de cet élément. En pratique, la longueur de diffusion cohérente $b_{\text {coh }}$ varie de façon aléatoire avec le numéro atomique.
La longueur de diffusion magnétique s'écrit d'autre part :

$$
p(Q)=\frac{r_{\mathrm{e}} \gamma}{2} \mu F_{\mathrm{M}}(Q)
$$

où $\gamma=1,91$ représente le rapport gyromagnétique du neutron,

$\mu$ le moment magnétique localisé sur chaque atome (mesuré en magnétons de Bohr),

$$
F_{\mathrm{M}}(Q)=\int \rho_{\mathrm{M}}(r) \mathrm{e}^{i \mathbf{Q} \mathbf{r}} \mathrm{d}_{3} \mathbf{r}
$$

le facteur de forme magnétique normalisé à 1 pour $Q=0$

et $\rho_{\mathbf{M}}(r)$ la densité d'aimantation atomique.

Ainsi, pour des neutrons polarisés dans l'état de spin $\pm \frac{1}{2}$ et un élément ferromagnétique, la longueur de diffusion neutronique $\left({ }^{1}\right)$ est donnée par la relation :

$$
f_{\mathrm{N}}^{ \pm}(Q)=b_{\text {coh }} \mp p(Q) \sin ^{2} \varphi
$$

où $\varphi$ désigne l'angle entre la polarisation et le vecteur de diffusion $\mathbf{Q}$.

1.1.3 Facteurs de structure partiels (FSP) d'un alliage binaire. - En généralisant la formule (1), on obtient la section efficace de diffusion d'un alliage binaire

$$
\begin{aligned}
\frac{\mathrm{d} \sigma}{\mathrm{d} \Omega}=C_{1} C_{2}\left(f_{1}-f_{2}\right)^{2} & + \\
& +\sum_{\alpha, \beta=1}^{2} C_{\alpha} C_{\beta} f_{\alpha} f_{\beta} A_{\alpha \beta}(Q)
\end{aligned}
$$

où $C_{\alpha}=N_{\alpha} / N$ et $f_{\alpha}$ représentent respectivement la concentration atomique et la longueur de diffusion du constituant $\alpha$ et les facteurs de structure partiels de Fournet [1], Faber-Ziman [2] (FFZ) $A_{\alpha \beta}(Q)$ décrivent les corrélations entre les diverses espèces chimiques.

Ces trois facteurs de structure $\left(A_{12}=A_{21}\right)$ sont reliés aux fonctions de corrélation partielles $P_{\alpha \beta}(r)$ par les équations :

$$
A_{\alpha \beta}(Q)=1+\rho \int\left[P_{\alpha \beta}(r)-1\right] \mathrm{e}^{i \mathbf{Q r}} \mathrm{d}_{3} \mathbf{r} .
$$

Par définition, $P_{\alpha \beta}(r)$ est la probabilité par unité de volume de trouver un atome du constituant $\alpha$ à une distance $r$ d'un autre atome $\beta$ (normalisée à 1 pour les grandes valeurs de $r$ ).

Bhatia et Thornton [3] préfèrent écrire la section efficace (6) sous la forme :

$$
\begin{aligned}
\frac{\mathrm{d} \sigma}{\mathrm{d} \Omega}=\bar{f}^{2} S_{\mathrm{NN}}(Q)+2 \bar{f} \Delta f C_{1} & S_{\mathrm{NC}}(Q)+ \\
& +C_{1} C_{2} \Delta f^{2} S_{\mathrm{CC}}(Q)
\end{aligned}
$$

( $\left.{ }^{1}\right)$ En toute rigueur $f_{\mathrm{N}}^{ \pm}$désigne la longueur de diffusion sans changement du spin neutronique $(+\rightarrow+$ ou $-\rightarrow-)$. 
qui fait intervenir la longueur de diffusion moyenne de l'alliage : $\bar{f}=C_{1} f_{1}+C_{2} f_{2}$, la différence des lon- gueurs de diffusion $\Delta f=f_{1}-f_{2}$ et les facteurs de structure :

$$
\left.\begin{array}{l}
S_{\mathrm{NN}}=1+\rho \int\left(C_{1}^{2} P_{11}+C_{2}^{2} P_{22}+2 C_{1} C_{2} P_{12}-1\right) \mathrm{e}^{i \mathbf{Q r}} \mathrm{d}_{3} r \\
S_{\mathrm{NC}}=\rho C_{2} \int\left[C_{1} P_{11}-C_{2} P_{22}+\left(C_{2}-C_{1}\right) P_{12}\right] \mathrm{e}^{i \mathbf{Q r}} \mathrm{d}_{3} r \\
S_{\mathrm{CC}}=1+\rho C_{1} C_{2} \int\left(P_{11}+P_{22}-2 P_{12}\right) \mathrm{e}^{i \mathbf{Q r}} \mathrm{d}_{3} r .
\end{array}\right\}
$$

Les trois facteurs de structure partiels de Bhatia et Thornton (BT) $S_{\mathrm{NN}}, S_{\mathrm{NC}}$ et $S_{\mathrm{CC}}$, qui sont des combinaisons linéaires des fonctions $\mathrm{A}_{\alpha \beta}$, caractérisent respectivement les corrélations densité-densité, densité-concentration et concentration-concentration. Ce formalisme permet de découpler les effets d'ordre géométrique $\left(S_{\mathrm{NN}}\right)$ des effets d'ordre chimique $\left(S_{\mathrm{CC}}\right)$ et convient particulièrement à l'étude des alliages de substitution (§ 2.4.2).

1.2 CHOIX D'UNE MÉTHODE DIFFRACTOMÉTRIQUE. 1.2.1 Electrons, rayons $\mathrm{X}$ et neutrons. - Si l'on ne désire qu'un seul diagramme de diffraction, le choix $\mathrm{du}$ rayonnement est dicté en premier lieu par l'épaisseur de l'échantillon disponible. En effet, les électrons, les rayons $\mathrm{X}$ cristallographiques et les neutrons couvrent des gammes d'épaisseur croissantes respectivement comprises entre $10^{-6}$ et $10^{-4} \mathrm{~cm}$ (couches minces obtenues par évaporation, sputtering...), $10^{-3}$ et $10^{-2} \mathrm{~cm}$ (échantillons préparés par trempe ultrarapide), $10^{-1}$ et $1 \mathrm{~cm}$ (métaux liquides ou amorphes massifs).

Pour étudier un amorphe massif, il est préférable d'utiliser les rayons $\mathrm{X}$ ou les neutrons. En effet, la diffraction électronique nécessite la préparation de couches minces qui peuvent avoir une structure différente de l'échantillon épais et donne des diagrammes dont l'analyse quantitative est délicate. Le choix entre rayons $\mathrm{X}$ et neutrons s'effectue alors en comparant les longueurs de diffusion $f_{\mathbf{X}}$ et $f_{\mathbf{N}}$. Les neutrons sont souvent mieux adaptés à l'étude des liquides et amorphes car ils offrent des avantages tels que :

- Sensibilité aux éléments légers et possibilité de distinguer des éléments de numéro atomique voisin.

- Sensibilité aux phénomènes magnétiques.

- Constance de la longueur de diffusion nucléaire facilitant les mesures aux grandes valeurs de $Q$.

- Souplesse en longueur d'onde donnant accès à une large gamme de $Q\left(10^{-3}\right.$ à $30 \AA^{-1}$ à l'Institut Laue Langevin).

1.2.2 Méthodes de détermination des facteurs de structure partiels d'un alliage binaire. - L'étude structurale complète d'un alliage binaire nécessite trois expériences de diffraction indépendantes. L'ensemble de ces mesures fournit un système d'équations linéaires $(\S 1.1 .3)$ à partir duquel sont calculés les trois facteurs de structure partiels. La précision de la méthode augmente avec le déterminant normalisé (ou pivot) du système [4]. Nous présentons ici les principales méthodes de détermination des FSP et leurs différents domaines d'application.

1.2.2.1 Méthode des concentrations. - La méthode dite des concentrations consiste à déterminer les FSP à partir de trois diagrammes de diffraction correspondant à des concentrations différentes. Comme elle suppose les FSP indépendants de la concentration, elle ne s'applique qu'aux très rares mélanges idéaux où les trois facteurs de structure partiels FFZ sont égaux entre eux [5] (§ 2.4.2.3).

1.2.2.2 Méthodes rigoureuses. $-a$ ) Méthode des trois rayonnements. - Les méthodes rigoureuses de détermination des FSP obtiennent trois équations indépendantes en faisant varier une ou deux longueurs de diffusion sans changer la concentration. Ce but peut être atteint en combinant trois rayonnements (X, électrons, neutrons) [6]. Toutefois, cette méthode délicate, qui utilise des échantillons de taille très inégale, n'a pas encore donné à notre connaissance de résultat quantitatif.

b) Méthodes de diffusion anomale. - En pratique, seules les méthodes basées sur l'emploi d'un rayonnement unique sont suffisamment précises. Parmi celles-ci, nous citerons tout d'abord les méthodes de diffusion anomale [7]. Mettant à profit les variations de l'amplitude de diffusion avec la longueur d'onde au voisinage d'une discontinuité d'absorption (rayons $\mathrm{X}$ ) ou d'une résonance de diffusion (neutrons), on obtient ici trois équations indépendantes en utilisant des longueurs d'onde différentes. Dans le cas des rayons $\mathrm{X}$, cette méthode pose toutefois des problèmes de précision car les variations relatives de l'amplitude de diffusion ne dépassent pas $20 \%$ [8], [9], [10]. Dans le cas des neutrons, aucune expérience exploitant ce principe n'a été publiée bien que les variations relatives de la longueur de diffusion soient 10 à 100 fois supérieures. D'après H. Egger [11], la nécessité de disposer d'un faisceau neutronique de longueur d'onde parfaitement définie et l'absorption considérable au voisinage 
de la résonance, constituent les obstacles majeurs à de telles mesures.

c) Méthode de substitution isotopique. - $\mathrm{La}$ méthode la plus précise, dite de substitution isotopique, a été introduite par Enderby, North et Egelstaff [12] et employée par de nombreux auteurs dans l'étude des sels fondus [13], [14], [4], [15] comme dans celle des alliages liquides [16]. On fait ici varier la longueur de diffusion nucléaire cohérente de l'un des constituants en changeant sa composition isotopique. Cette méthode est applicable en principe à tous les systèmes dont l'un des constituants possède plusieurs isotopes. Elle peut toutefois soulever des problèmes financiers si la préparation de l'alliage exige une quantité importante d'un isotope.

d) Méthodes des neutrons polarisés. - La méthode des neutrons polarisés [17], [18] qui fait varier la longueur de diffusion magnétique des neutrons en changeant l'orientation relative de la polarisation, de l'aimantation et du vecteur de diffusion, ne s'applique qu'aux alliages ferro- ou ferrimagnétiques amorphes. On obtient ici trois équations indépendantes en enregistrant deux diagrammes de diffraction avec des polarisations $\pm \mathbf{P}$ perpendiculaires au vecteur de diffusion $\mathbf{Q}$ et un diagramme avec la polarisation parallèle à $\mathbf{Q}$ (voir équation (5)).

Cette technique, qui utilise un seul rayonnement et un seul échantillon, reste précise tant que $Q$ est inférieur à $10 \AA^{-1}$. Au-delà de cette limite, les longueurs de diffusion magnétiques deviennent trop petites par rapport aux longueurs de diffusion nucléaires et le système est indéterminé. Cette restriction n'est pas gênante toutefois, puisque les phénomènes d'ordre ou de ségrégation dans les alliages amorphes se manifestent pour des valeurs de $Q$ inférieures à quelques $\AA^{-1}(\S 2.4 .2 .4)$.

1.2.2.3 Méthode des deux rayonnements. La méthode des deux rayonnements introduite par Ruppersberg et Egger [19], [20], [21] s'applique en toute rigueur aux alliages de substitution où les constituants ont le même diamètre atomique. Dans ce cas, il n'y a pas de corrélation entre densité et concentration, la fonction $S_{\mathrm{NC}}$ est nulle et il suffit d'une mesure de rayons $X$ et d'une mesure de neutrons pour déterminer les deux facteurs de structure partiels $S_{\mathrm{NN}}$ et $S_{\mathrm{CC}}$. Cette réduction du nombre des inconnues entraîne un gain considérable de précision.

Par ailleurs, si les constituants ont des tailles différentes, l'approximation $S_{\mathrm{NC}} \simeq 0$ reste valable tant que l'écart relatif des diamètres atomiques ne dépasse pas $5 \%$ [18]. Elle peut donc être utilisée dans de nombreux cas où l'on dispose seulement de deux diagrammes de diffraction.

2. Modèles structuraux géométriques. - 2.1 LIQUIDES ET AMORPHES. - Un objectif essentiel des théories de l'état liquide consiste à déterminer les facteurs de structure des métaux et alliages à partir des potentiels d'interaction de paire.

Ce problème a été abordé par des calculs analytiques fondés sur l'approximation de Percus-Yevick [22] et l'utilisation de potentiels de sphères dures [23], [24] ou de sphères adhésives [25]. Mais ces théories ont surtout donné des résultats satisfaisants dans l'étude des propriétés thermodynamiques.

Les calculs numériques de dynamique moléculaire [26], [27] permettent d'obtenir les facteurs de structure en résolvant les équations du mouvement de quelques centaines de particules en interaction. Cette méthode a donné d'excellents résultats dans le cas des métaux liquides [28] comme dans celui des sels fondus [29].

Cependant, malgré des analogies structurales remarquables, ces théories ne peuvent être directement appliquées aux métaux et alliages amorphes car elles supposent toutes que le système est en équilibre thermodynamique. Cela explique la floraison et l'intérêt des modèles phénoménologiques d'états amorphes métastables.

Ainsi, le modèle de l'atome entouré (J. C. Mathieu [30], [31], [32]) et celui des translateurs-vibrateurs (P. Hicter [33], [34]) qui ont permis d'interpréter de nombreux résultats expérimentaux sur les métaux et alliages liquides, sont d'un grand secours dans l'étude des amorphes.

Les modèles géométriques que nous présentons ici, en sont l'équivalent structural. Comme ils assimilent les atomes à des sphères adhésives, ils ne s'appliquent qu'aux alliages liquides ou amorphes dont les diamètres atomiques sont additifs.

2. 2 MÉTAUX ET SEMI-CONDUCTEURS AMORPHES. Une fois écartée l'hypothèse microcristalline (J. Dixmier [35]), la structure des métaux et alliages amorphes a été simulée à l'aide d'amá (ou agrégats) de sphères compacts et désordonnés où chaque sphère possède environ dix premières voisines (voir $\S 2.3 .3$ et 2.3.6).

Toutefois, comme ces modèles excluent tout effet de liaison directionnel, ils ne conviennent pas aux amorphes covalents. Ainsi, pour représenter la structure des semi-conducteurs tétravalents, il faut utiliser le modèle peu dense de D. E. Polk [36] où les angles de liaison restent proches de $109^{\circ}$ et le nombre de premiers voisins égal à 4 .

Nous nous consacrerons désormais aux modèles géométriques qui concernent les métaux et alliages amorphes.

\subsection{MODÈLES GÉOMÉTRIQUES DE MÉTAUX AMORPHES.} - 2.3.1 Géométrie des réseaux amorphes. - De nombreux agrégats de sphères adhésives ont été construits pour simuler la structure des métaux amorphes [37], [38], [39], [40], [41]. Tous ces agrégats sont générés en faisant croître un germe de trois ou quatre sphères par additions successives de nouvelles sphères $P_{i}$ tangentes à trois sphères $A_{i}, B_{i}$ et $C_{i}$ de 
l'amas. Le centre de chaque sphère $P_{i}$ forme ainsi avec le centre de ses trois premières voisines un tétraèdre $A_{i} B_{i} C_{i} P_{i}$ plus ou moins régulier. Un réseau amorphe peut donc être caractérisé par la forme moyenne de ces tétraèdres que décrit le paramètre :

$K=\frac{1}{N} \sum_{i=1}^{N} \frac{A_{i} B_{i}+B_{i} C_{i}+C_{i} A_{i}+3 d}{6 d}(\geqslant 1)$

où $N$ désigne le nombre des sphères et $d$ leur diamètre.

Le réseau amorphe correspondant à $K=1$ est appelé réseau polytétraédrique parfait $\left({ }^{2}\right)$.

2.3.2 Réseau compact amorphe. - Un agrégat amorphe est aussi caractérisé par sa compacité :

$$
\gamma=\frac{N v}{V}=\rho v
$$

où $v=\pi d^{3} / 6$ désigne le volume d'une sphère et $V$ celui de l'agrégat.

$\gamma$ est une fonction croissante de $K$. En l'absence de démonstration mathématique, on peut estimer la valeur maximum de $\gamma$ à l'aide des mesures effectuées par Finney [38] sur des amas de billes d'acier ou des calculs numériques d'Ichikawa [40].

On trouve alors : $\gamma_{\max } \simeq 0,63 \pm 0,01$.

La valeur correspondante de $K$, calculée par une extrapolation linéaire des résultats que nous avons obtenus [18] dans l'intervalle :

$$
0,44(K=1) \leqslant \gamma \leqslant 0,57(K=1,15),
$$

est voisine de $: K_{\max } \simeq 1,22$.

Nous appellerons réseau compact amorphe, œ réseau de compacité et de désordre maximums.

2.3.3 Nombre de coordination. - Dans un modèle géométrique, les sphères au contact sont représentées par un pic $\delta(r-d)$ sur la fonction $P(r)$. On peut donc définir le nombre de coordination (moyen) par la relation :

$$
\eta=\rho \int_{0}^{d+\varepsilon} P(r) 4 \pi r^{2} \mathrm{~d} r
$$

où $\varepsilon$ est très petit devant $d$.

Les calculs montrent que $\eta$ croît de 6,5 à 7,1 lorsque $\gamma$ varie de 0,44 à 0,63 [40], [18].

2.3.4 Facteur de structure des réseaux amorphes monoatomiques. - Le facteur de structure d'un agrégat amorphe contenant plus de 1000 sphères est pratiquement indépendant de $N$ [41]. Les facteurs de structure des différents réseaux amorphes varient donc essentiellement avec $\gamma$ (ou $K$ ) (Fig. 1).

Ainsi, la valeur du facteur de structure pour $Q=0$ décroît lorsque $\gamma$ augmente. Une extrapolation linéaire

( ${ }^{2}$ Il ne faut pas confondre ce réseau dont le nombre de coordination moyen est voisin de 10 , avec le réseau tétracoordonné de Polk [36]!

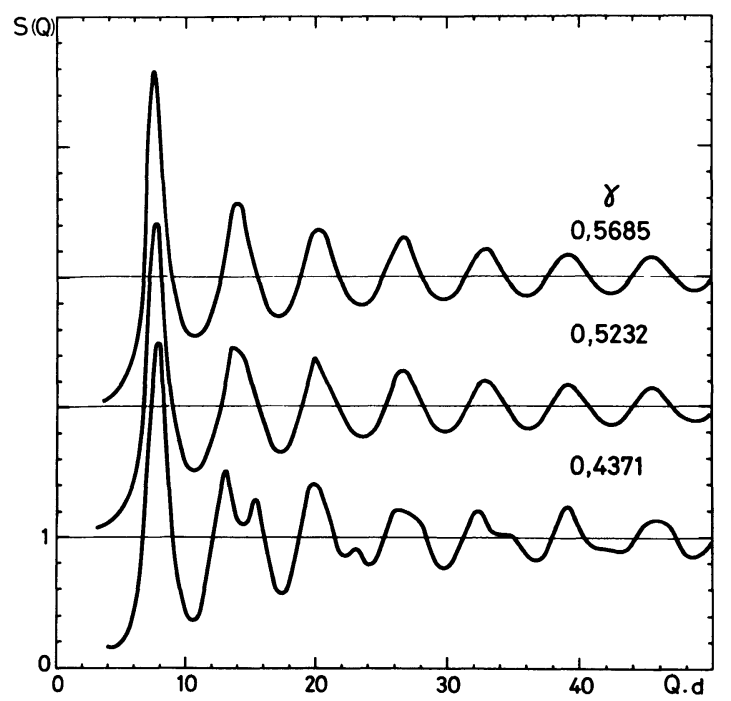

Fig. 1. - Variations du facteur de structure avec la compacité dans un amorphe monoatomique.

[Variations of the structure factor with packing fraction in monoatomic glasses.]

des calculs effectués dans l'intervalle $0,44 \leqslant \gamma \leqslant 0,57$ conduit à $S(0) \simeq 0$ pour $\gamma \simeq \gamma_{\max }$ ! [18]. Ces résultats sont bien naturels puisque $S(0)$ est proportionnelle aux fluctuations quadratiques moyennes de densité ou encore à la compressibilité du réseau amorphe [42].

D'autre part, la position $Q_{1}$ du premier maximum de $S(Q)$ est très voisine de la valeur

$$
Q_{111}=2 \pi \frac{\sqrt{3}}{\sqrt{2}} \frac{1}{d}=\frac{7,695}{d}
$$

qui correspond à une réflexion sur les plans d'empilement compact 111 d'un réseau cubique à faces centrées. C'est pourquoi nous désignons par le terme de «pseudo-période » la distance :

$$
d_{1}=\frac{2 \pi}{Q_{1}} \simeq d_{111}=d \frac{\sqrt{2}}{\sqrt{3}} .
$$

Lorsque $K$ varie de 1 à $K_{\max } Q_{1}$ décroît légèrement de 7,695 $d$ à 7,45 $d$ [40], [18]. Par ailleurs, l'intensité $S\left(Q_{1}\right)$ de ce premier maximum augmente avec $K$ depuis $S\left(Q_{1}\right) \simeq 2,33$ pour le réseau polytétraédrique parfait jusqu'à $S\left(Q_{1}\right) \simeq 2,7$ pour le réseau compact amorphe [40].

La forme révélatrice du deuxième pic de $S(Q)$ a suscité depuis longtemps l'intérêt des spécialistes [35]. Dans le cas du réseau polytétraédrique parfait, ce pic est séparé en deux maximums distincts. Le plus intense est situé en $Q_{2}^{\prime} \simeq 13,2 / d$ et le second en $Q_{2}^{\prime \prime} \simeq 15,2 / d$. Lorsque $\gamma$ augmente, ces deux maximums se rapprochent. Pour $\gamma \simeq 0,52$ on ne distingue plus qu'un épaulement sur un pic large. Dans le cas du réseau compact amorphe, ce pic est pratiquement symétrique et situé en $Q_{2} \simeq 14,0 / d$.

Enfin, le pic $\delta(r-d)$ qui correspond aux premiers 
voisins dans la fonction $P(r)$ détermine la forme asymptotique de $S(Q)$. Il vient ainsi :

$\lim _{Q \rightarrow \infty} S(Q)=1+\eta \frac{\sin (Q d)}{Q d}$ pour $K \neq 1\left(^{3}\right)$.

Inversement, il est possible de calculer le nombre de coordination $\eta$ à partir de l'amplitude des oscillations de $S(Q)$ aux grands $Q$.

2.3.5 Distributions aléatoires de lacunes. - Afin de pouvoir comparer les résultats précédents aux facteurs de structure expérimentaux, nous rappellerons successivement l'influence des lacunes et des vibrations atomiques sur la fonction $S(Q)$.

L'introduction de $N_{1}$ lacunes sur les $N$ sites d'un réseau amorphe change le nombre d'atomes par unité de volume en :

$$
\rho_{1}=\frac{N-N_{1}}{N} \rho
$$

mais elle ne modifie pas la fonction $P(r)$ [41].

En conséquence, le facteur de structure devient :

$$
S_{1}(Q)=1+\frac{\rho_{1}}{\rho}[S(Q)-1] .
$$

2.3.6 Vibrations atomiques. - Si l'on suppose que les atomes vibrent à la fréquence $v$ (modèle d'Einstein) autour des positions d'équilibre (métastable) du réseau amorphe, la fonction de distribution de paire devient [5] :

$$
P_{v}(r)=\left(4 \pi \sigma^{2}\right)^{-3 / 2} \int P\left(\mathbf{r}-\mathbf{r}^{\prime}\right) \exp \left(-\frac{r^{\prime 2}}{4 \sigma^{2}}\right) \mathrm{d}_{3} r^{\prime}
$$

et le déplacement quadratique moyen s'écrit :

$$
\sigma^{2}=\frac{h}{8 \pi^{2} m v} \operatorname{coth}\left(\frac{h v}{2 k_{\mathrm{B}} T}\right)
$$

où $h$ désigne la constante de Planck, $k_{\mathrm{B}}$ la constante de Boltzmann, $T$ la température et $m$ la masse atomique.

L'élargissement du pic des premiers voisins correspondant à l'équation de convolution (15) exige une redéfinition du nombre de coordination. De nombreuses solutions ont été proposées. Nous préférons adopter la définition :

$$
\eta^{\prime}=2 \rho \int_{0}^{d} P_{v}(r) 4 \pi r^{2} \mathrm{~d} r
$$

$\eta^{\prime}$ est supérieur à $\eta$ car des voisins qui ne sont pas au contact dans le réseau amorphe de sphères adhésives se trouvent inclus dans cette définition. Pour la

$\left({ }^{3}\right)$ Ce résultat n'est pas valable pour le réseau polytétraédrique parfait dont la fonction $P(r)$ présente plusieurs pics de Dirac. plupart des métaux liquides au voisinage du point de fusion $\eta^{\prime} \simeq 10$.

Enfin, les vibrations atomiques entraînent l'apparition d'un facteur de Debye-Waller dans l'expression du facteur de structure :

$$
S_{v}(Q)=1+\exp \left(-\sigma^{2} Q^{2}\right)[S(Q)-1] .
$$

2.4 Modèles D'Alliages BINAIRES AMORPHES. 2.4.1 Ordres chimiques et géométriques. - Pour représenter la structure d'un alliage binaire amorphe par un modèle de sphères adhésives, il faut définir :

- le rapport des diamètres atomiques $\delta=\frac{d_{2}}{d_{1}}(\geqslant 1)$,

- la concentration $C_{1}=1-C_{2}$,

- une règle d'empilement géométrique $(\S 2.3 .1)$ et une règle d'empilement chimique des sphères.

On peut ainsi distinguer les alliages ordonnés et ségrégés correspondant à des attractions hétéro- ou homoatomiques et les alliages désordonnés.

Nous étudierons tout d'abord les phénomènes d'ordre chimique pur dans le cas des alliages de substitution pour lesquels une description analytique à l'aide du paramètre d'ordre des premiers voisins est possible [5]. Nous montrerons ensuite comment les phénomènes d'ordre chimique se couplent avec les effets de taille dans les alliages où $\delta$ diffère de 1 [5].

2.4.2 Alliages de substitution $(\delta=1)$ - 2.4.2.1 Fonctions de distribution partielles de paire. Par définition, les deux constituants d'un alliage de substitution ont le même nombre de voisins quelle que soit la distance $r\left({ }^{4}\right)$. En termes analytiques, les fonctions de distribution partielles de paire vérifient les équations :

$$
\begin{aligned}
C_{1} P_{11}(r)+C_{2} P_{12}(r)= & C_{2} P_{22}(r)+ \\
& +C_{1} P_{21}(r)=P_{\gamma}(r)
\end{aligned}
$$

où $P_{\gamma}(r)$ désigne la fonction de distribution de paire du réseau amorphe de compacité $\gamma$ formé par tous les atomes (indépendamment de leur nature chimique). En conséquence la compacité maximum d'un alliage de substitution est identique à celle d'un amorphe monoatomique.

2.4.2.2 Facteurs de structure partiels de Bhatia et Thornton et paramètre d'ordre. - Dans un alliage de substitution, il est possible de changer la concentration sans changer la densité locale en permutant un atome de type 1 et un atome de type 2. Cette absence de corrélation entre densité et concentration se traduit par la relation (équivalente à l'équation (17)) :

$$
S_{\mathrm{NC}}(Q)=0 \text {. }
$$

$\left({ }^{4}\right)$ Cette définition exige donc l'égalité des diamètres atomiques et exclut tout effet de liaison directionnel (§ 2.2). 
D'autre part, la fonction $S_{\mathrm{NN}}$ s'identifie au facteur de structure d'un amorphe monoatomique :

$$
S_{\mathrm{NN}}(Q)=1+\rho \int\left[P_{v}(r)-1\right] \mathrm{e}^{i \mathbf{Q r}} \mathrm{d}_{3} r=S_{\gamma}(Q) .
$$

Ce facteur de structure géométrique qui est indépendant de la concentration et de l'ordre chimique a déjà été étudié au paragraphe 2.3.4.

C'est pourquoi nous nous consacrerons désormais à la fonction $S_{\mathrm{CC}}$ qui décrit l'ordre chimique.

Dans un modèle de sphères adhésives, les pics des premiers voisins sont représentés par des fonctions $\delta(r-d)$ et déterminent le comportement asymptotique des fonctions $S_{\mathrm{NN}}$ et $S_{\mathrm{CC}}$. Désignons en effet par $\eta_{12}$ le nombre de sphères de type 2 en contact avec une sphère de type 1 (ou nombre de coordination partiel 1-2) et $\eta_{1}=\eta_{11}+\eta_{12}$ le nombre total de sphères en contact avec une sphère de type 1 (ou nombre de coordination 1). $\eta_{1}$ et $\eta_{2}$ étant égaux, on peut définir le paramètre d'ordre des premiers voisins :

$$
\xi=1-\frac{\eta_{12}}{\eta_{1}} \frac{1}{C_{2}}=1-\frac{\eta_{21}}{\eta_{2}} \frac{1}{C_{1}} .
$$

Il vient alors :

$$
\begin{aligned}
& \lim _{Q \rightarrow \infty} S_{\mathrm{NN}}(Q)=1+\eta \frac{\sin (Q d)}{Q d} \\
& \lim _{Q \rightarrow \infty} S_{\mathrm{CC}}(Q)=1+\xi \eta \frac{\sin (Q d)}{Q d} .
\end{aligned}
$$

Selon que l'alliage est ségrégé $(\xi>0)$ ou ordonné $(\xi<0)$ les oscillations de $S_{\mathrm{NN}}$ et $S_{\mathrm{CC}}$ aux grands $Q$ sont donc en phase ou en opposition de phase. D'un point de vue pratique, le rapport de l'amplitude des oscillations de $S_{\mathrm{CC}}$ et $S_{\mathrm{NN}}$ permet d'obtenir la valeur du paramètre d'ordre des premiers voisins car il est insensible aux vibrations atomiques.

Les formes asymptotiques (21) constituent une bonne approximation tant que $Q$ est supérieur à $15 / d$ [5]. Pour des valeurs de $Q$ inférieures, la fonction $S_{\mathrm{CC}}$ dépend de l'ordre chimique à plus grande distance et doit être calculée numériquement à l'aide des modèles de sphères adhésives.

Les trois paragraphes suivants traitent ce problème.

2.4.2.3 Mélanges idéaux $(\delta=1, \xi=0)$. - Dans un alliage désordonné de substitution les trois fonctions $P_{\alpha \beta}(r)$ sont égales à la fonction de distribution de paire de l'amorphe global :

on a donc :

$$
P_{11}(r)=P_{12}(r)=P_{22}(r)=P_{\gamma}(r)
$$

$$
\begin{gathered}
S_{\mathrm{CC}}(Q)=1 \\
\text { ou encore : } \\
A_{11}(Q)=A_{12}(Q)=A_{22}(Q)=S_{\gamma}(Q) .
\end{gathered}
$$

C'est le seul cas où lés trois facteurs de structure partiels sont indépendants de la concentration. La méthode de détermination des FSP dite des concentrations est donc soumise aux conditions expresses : $\delta=1, \xi=0$. C'est pourquoi elle est généralement incorrecte.

2.4.2.4 Alliages ordonnés de substitution $(\delta=1, \xi<0)$. - a) Propriétés communes. - Dans un alliage ordonné il existe des groupes de sphères formés d'une sphère centrale de type 1 (ou 2) entourée préférentiellement de sphères de type 2 (ou 1). Le diamètre moyen de ces " atomes entourés " [30] est approximativement égal à :

$$
d_{\mathrm{s}} \simeq \frac{2 \sqrt{2}}{\sqrt{3}} d
$$

L'empilement de ces atomes entourés donne naissance à une nouvelle pseudo-période ( $§ 2.3 .4$ ) chimique :

$$
Q_{\mathrm{s}} \simeq 2 \pi \frac{\sqrt{3}}{\sqrt{2}} \frac{1}{d_{\mathrm{s}}}
$$

qui se traduit par un maximum de surstructure dans la fonction $S_{\mathrm{CC}}(Q)$. La position de ce prépic est reliée à la position $Q_{1} \simeq 2 \pi \frac{\sqrt{3}}{\sqrt{2}} \frac{1}{d}(\$ 2.4 .2 .2$ et 2.3 .4$)$ du premier pic de $S_{\mathrm{NN}}$ par l'équation :

$$
Q_{\mathrm{s}} \simeq \frac{\sqrt{3}}{2 \sqrt{2}} Q_{1} \simeq 0,61 Q_{1}
$$

et son intensité $S_{\mathrm{CC}}\left(Q_{\mathrm{s}}\right)$ croît avec $|\xi|$ (voir Tableau I et Fig. 2).
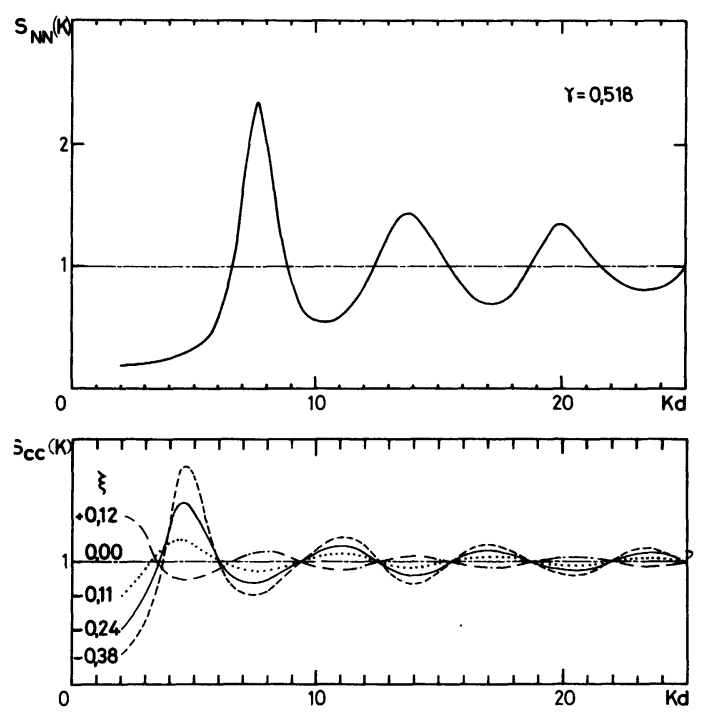

Fig. 2. - Variations des facteurs de structure BT avec le paramètre d'ordre dans les alliages de substitution.

[Variations of the Bhatia-Thornton partial structure factors with order parameter in substitutionnal alloys.] 
Tableau I. - Variations de l'intensité des deux premiers extremums de $S_{\mathrm{CC}}$ dans les alliages de substitution (pour $\gamma=0,52$ ).

$\begin{array}{crcrrrrr}\xi & -0,391 & -0,381 & -0,236 & -0,110 & 0 & 0,120 & 0,116 \\ C_{1} & 0,500 & C_{\max }=0,287 & 0,200 & 0,100 & 0 \leqslant C_{1} \leqslant 1 & 0,300 & 0,500 \\ S_{\mathrm{cc}}\left(Q_{\mathrm{s}}\right)-1 & 0,869 & 0,784 & 0,476 & 0,182 & 0 & -0,140 & -0,159 \\ S_{\mathrm{cc}}\left(Q_{1}\right)-1 & -0,279 & -0,288 & -0,181 & -0,079 & 0 & 0,077 & 0,093\end{array}$

La valeur du paramètre d'ordre des premiers voisins suffit donc à déterminer la fonction $S_{\mathrm{cc}}(Q)$ d'un alliage ordonné de substitution pour toutes les valeurs de $Q$.

b) Ordre maximum. - Dans un alliage à ordre maximum, les sphères 1 sont uniquement entourées de sphères 2 et le paramètre d'ordre des premiers voisins est égal à :

$$
\xi_{\min }=-\frac{C_{1}}{1-C_{1}} .
$$

Quelle que soit la concentration, $\xi$ est donc supérieur ou égal à $\xi_{\min }$. Cependant, $\xi$ ne peut atteindre la valeur $\xi_{\min }$ que dans un intervalle de concentration limité $0 \leqslant C_{1} \leqslant C_{\max }$. En effet, il est impossible de construire un réseau amorphe (à ordre maximum) de sphères 2 contenant des trous isolés entre eux pour accommoder les sphères $1\left({ }^{5}\right)$ au-delà d'une concentration $C_{\max } \simeq 0,29$ correspondant à un nombre de coordination partiel $: \eta_{22}^{\min } \simeq 4,0[5]$.

2.4.2.5 Alliages ségrégés de substitution $(\delta=1, \xi>0)$. - Si la ségrégation des atomes de même nature chimique est limitée aux premiers voisins, elle peut être décrite à l'aide du paramètre $\xi$. $\mathrm{La}$ structure de ces alliages ségrégés se déduit alors de celle des alliages ordonnés en changeant le signe de $\xi$. La fonction $S_{\mathrm{CC}}$ présente donc en $Q_{\mathrm{s}}$ un préminimum de surstructure dont la profondeur augmente avec $\xi$ (voir Tableau I et Fig. 2) ([5], [32]).

Si la ségrégation se développe au-delà des premiers voisins, le paramètre $\xi$ ne suffit plus à décrire l'ordre chimique. En l'absence de modèle, on peut cependant penser que la fonction $S_{\mathrm{CC}}$ présente encore un préminimum de surstructure qui se déplace vers les petits $Q$ lorsque la ségrégation s'étend (c'est-à-dire lorsque la pseudo-période chimique augmente).

2.4.2.6 Formalisme de Fournet-Faber-Ziman. L'utilisation du formalisme de FFZ pour décrire la structure des alliages de substitution conduit à des résultats plus complexes. En effet, les trois fonctions $A_{11}, A_{22}$ et $A_{12}$ sont affectées par les phénomènes d'ordre ou de ségrégation et présentent en $Q_{\mathrm{s}}$ des prépics ou des préminimums de surstructure [5], [32].

2.4.3 Alliages à effet de taille $(\delta \neq 1) .-2.4 .3 .1$ Volume de mélange. - Quand les constituants ont des diamètres différents, la compacité maximum de l'alliage doit être supérieure à celle d'un amorphe

$\left({ }^{5}\right)$ Ce problème est donc un problème de percolation. monoatomique car des trous de taille infraatomique dans le réseau des grandes sphères peuvent être comblés par de petites sphères [37]. Toutefois nos calculs et les mesures de volume effectuées par C. Lemaignan [43] indiquent que cet effet est négligeable dans l'intervalle : $1 \leqslant \delta \lesssim 1,35$. Comme cet intervalle couvre une large gamme d'alliages, il semble qu'il faille attribuer la plupart des volumes de mélange mesurés dans les liquides à des effets de liaison chimique et non à ces effets géométriques.

2.4.3.2 Attroupements. - Les effets de taille dépendent évidemment du nombre (entier) maximum $n$ de sphères de diamètre $d_{1}$ qui peuvent s'agglutiner autour d'une sphère de diamètre $d_{2} . n$ croît avec $\delta$ en suivant une courbe en escalier dont les discontinuités ont été calculées par Schütte et Van Der Waerden [44]. Dans l'intervalle $0,5 \leqslant \delta \leqslant 1,5$ la courbe de Schütte est bien représentée par l'approximation linéaire [5] :

$$
n(\delta) \simeq 12 \delta-1
$$

où $n$ n'est plus un entier.

En présence d'effets de taille, il n'est donc plus possible de définir simplement le paramètre d'ordre des premiers voisins car les nombres de coordination de chaque constituant sont différents et la relation (20) n'est plus vérifiée.

2.4.3.3 Alliages désordonnés. - L'étude des alliages désordonnés où les sphères sont mélangées au hasard est révélatrice des seuls effets de taille.

Dans ce cas les sphères 2 (1) sont équivalentes à des lacunes distribuées au hasard dans le réseau amorphe des sphères 1 (2) (\$2.3.5). Toutefois, le diamètre $d_{2}\left(d_{1}\right)$ de ces lacunes étant différent de $d_{1}\left(d_{2}\right)$, les fonctions de distribution partielles de paire dépendent de la concentration et du rapport des diamètres atomiques.

C'est pourquoi, lorsque $\delta-1$ augmente, les premiers maximums des facteurs de structure FFZ se déplacent et deviennent de plus en plus asymétriques (Fig. 3). Pour $\gamma=0,54$ et $1 \leqslant \delta \leqslant 1,25$, les calculs numériques [5] montrent que la position de ces maximums vérifie la relation phénoménologique :

$$
Q_{1}^{\alpha \beta}=\frac{1}{d_{\alpha \beta}}\left[7,6-4,3\left(\frac{\bar{d}}{d_{\alpha \beta}}-1\right)\right]
$$

où $\bar{d}=C_{1} d_{1}+C_{2} d_{2}$ désigne le diamètre moyen des sphères et $d_{\alpha \beta}=\frac{d_{\alpha}+d_{\beta}}{2}$. Par ailleurs, la forme 

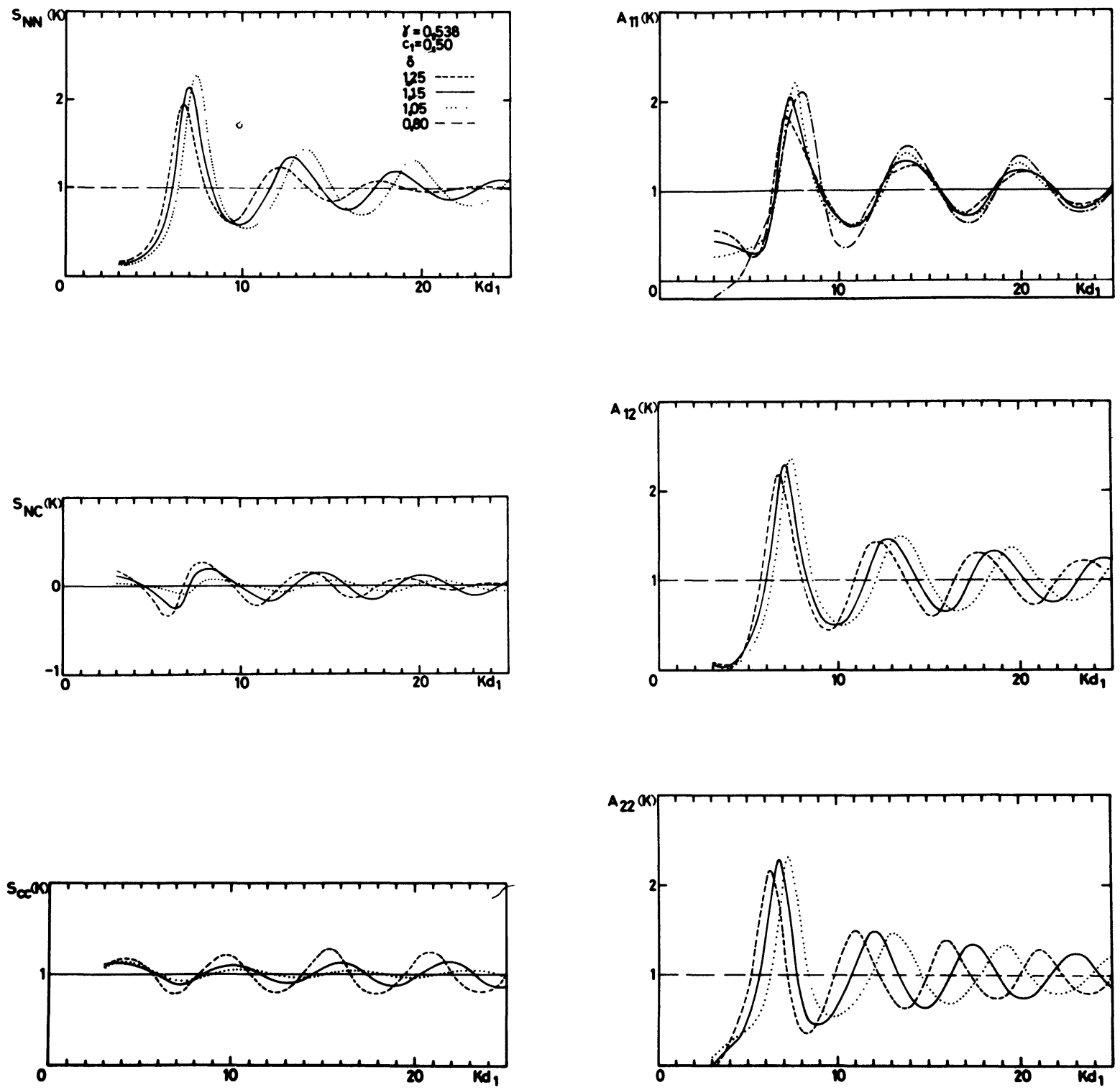

Fig. 3. - Variations des facteurs de structure BT et FFZ avec le rapport des diamètres atomiques dans les alliages désordonnés.

[Variations of the Bhatia-Thornton and Fournet-Faber-Ziman partial structure factors with atomic diameter ratio in disordered alloys.]

asymptotique des facteurs de structure FFZ est déterminée par l'intensité des pics $\delta\left(r-d_{\alpha \beta}\right)$ qui représentent les premiers voisins au contact dans les fonctions $P_{\alpha \beta}$ (formule (7)). Il vient ainsi :

$$
\lim _{Q \rightarrow \infty} A_{\alpha \beta}=1+\frac{\eta_{\alpha \beta}}{C_{\beta}} \frac{\sin \left(Q d_{\alpha \beta}\right)}{Q d_{\alpha \beta}} .
$$

Inversement, les relations (25) ou (26) permettent de calculer les distances $d_{\alpha \beta}$ à partir de la position du premier pic ou du comportement asymptotique des fonctions $A_{\alpha \beta}$. On peut alors vérifier si l'alliage satisfait à la loi d'additivité des diamètres atomiques :

$$
d_{12}=\frac{1}{2}\left(d_{11}+d_{22}\right)=\frac{1}{2}\left(d_{1}+d_{2}\right) .
$$

Examinons l'influence de l'effet de taille sur les facteurs de structure BT d'un alliage désordonné.
Lorsque $\delta$ s'écarte de 1 , des oscillations d'amplitude croissante apparaissent dans les fonctions $S_{\mathrm{NC}}$ et $S_{\mathrm{CC}}$ (Fig. 4). (Toutefois, $S_{\mathrm{CC}}$ ne présente pas de prépic intense de surstructure comme dans le cas des alliages ordonnés (§ 2.4.3.4).) Par ailleurs, le premier maximum de la fonction $S_{\mathrm{NN}}$ se déplace conformément à l'équation :

$$
Q_{1} \simeq \frac{7,6}{\bar{d}} \quad(\text { pour } \gamma=0,54)
$$

(qui résulte des $\S 2.3 .4$ et 2.4.3.2).

Enfin, les facteurs de structure BT présentent aux grands $Q$ des battements entre trois termes du type :

$$
\frac{\sin \left(Q d_{\alpha \beta}\right)}{Q d_{\alpha \beta}} \text { (voir formules (9) et (26)) . }
$$



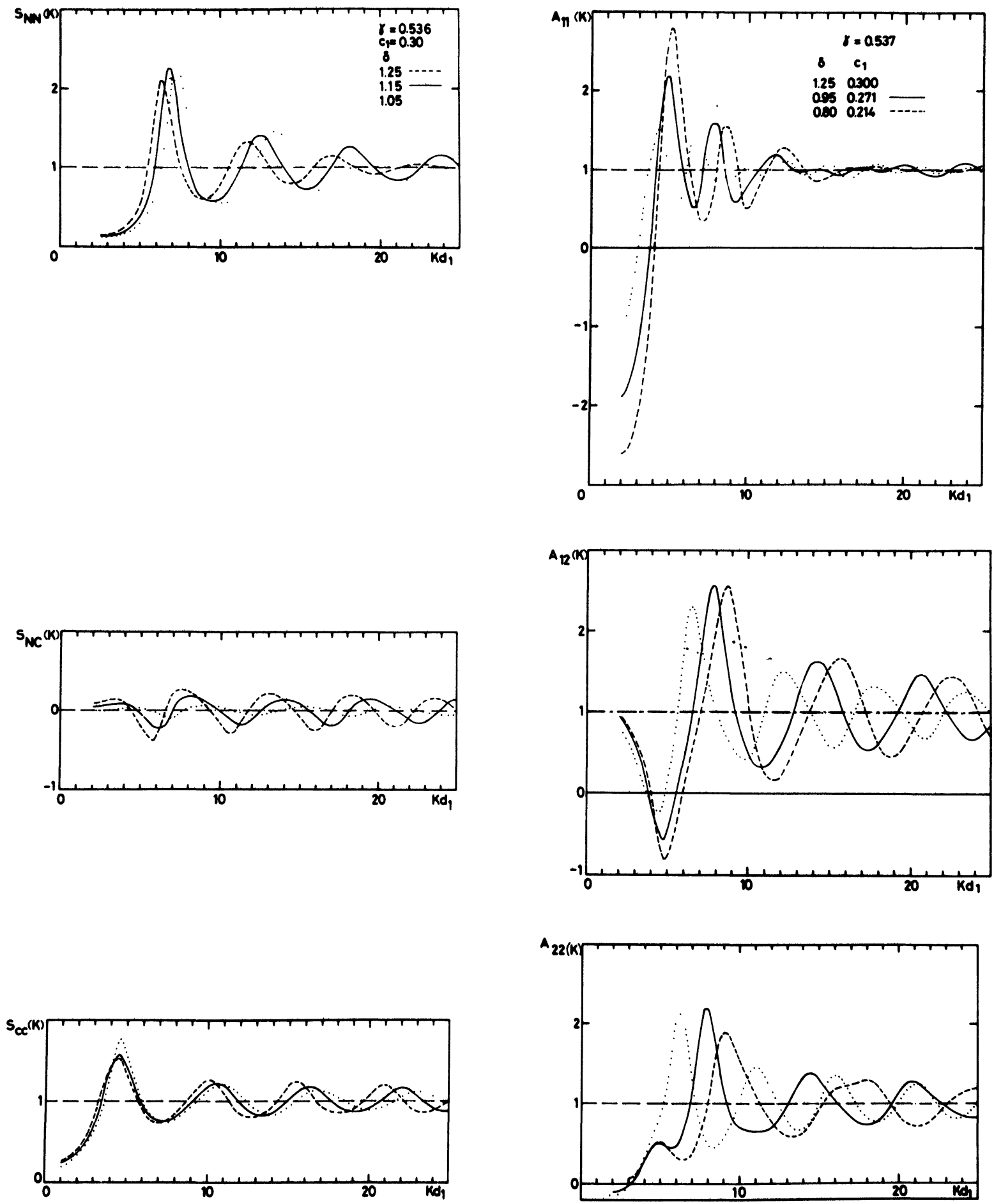

Fig. 4. - Variations des facteurs de structure BT et FFZ avec le rapport des diamètres atomiques dans les alliages à ordre maximum.

[Variations of the Bhatia-Thornton and Fournet-Faber-Ziman partial structure factors with atomic diameter ratio in maximum order alloys.]

2.4.3.4 Alliages ordonnés. - a) Comportement général. - Afin de mettre en évidence les effets d'ordre chimique, nous comparerons les facteurs de structure BT des alliages ordonnés avec ceux des alliages désordonnés pour une même valeur de $\delta$.

L'apparition d'un prépic de surstructure dans la fonction $S_{\mathrm{CC}}$ constitue le signe principal des phénomènes d'ordre (Fig. 4). La position relative de ce prépic par rapport au premier pic de $S_{\mathrm{NN}}$ est quasiment indépendante de $\delta$ (pour $1 \leqslant \delta \leqslant 1,25$ ) et suit l'équation (23).

b) Ordre maximum. - Il est impossible de construire un réseau amorphe à ordre maximum (dans lequel les sphères 1 sont uniquement entourées de sphères 2) si la concentration $C_{1}$ est supérieure à $C_{\max }$. La concentration $C_{\max }$ augmente avec $\delta$ mais 
elle correspond toujours à un nombre de coordination partiel $\eta_{22}^{\min }$ voisin de 4 (voir Tableau II).

\section{Tableau II.}

$\begin{array}{lllll}\delta & 1 & 1,05 & 1,15 & 1,25 \\ C_{\max } & 0,287 & 0,315 & 0,356 & 0,396 \\ \eta_{22}^{\min } & 4,01 & 3,92 & 3,96 & 4,02\end{array}$

3. Application aux alliages cobalt-phosphore. 3.1 RÉSULTATS EXPÉRIMENTAUX. - La comparaison entre les FSP mesurés et calculés constitue un test crucial pour les modèles. Malheureusement, les résultats publiés [45], [17], [9], [46] sont rares. Nous
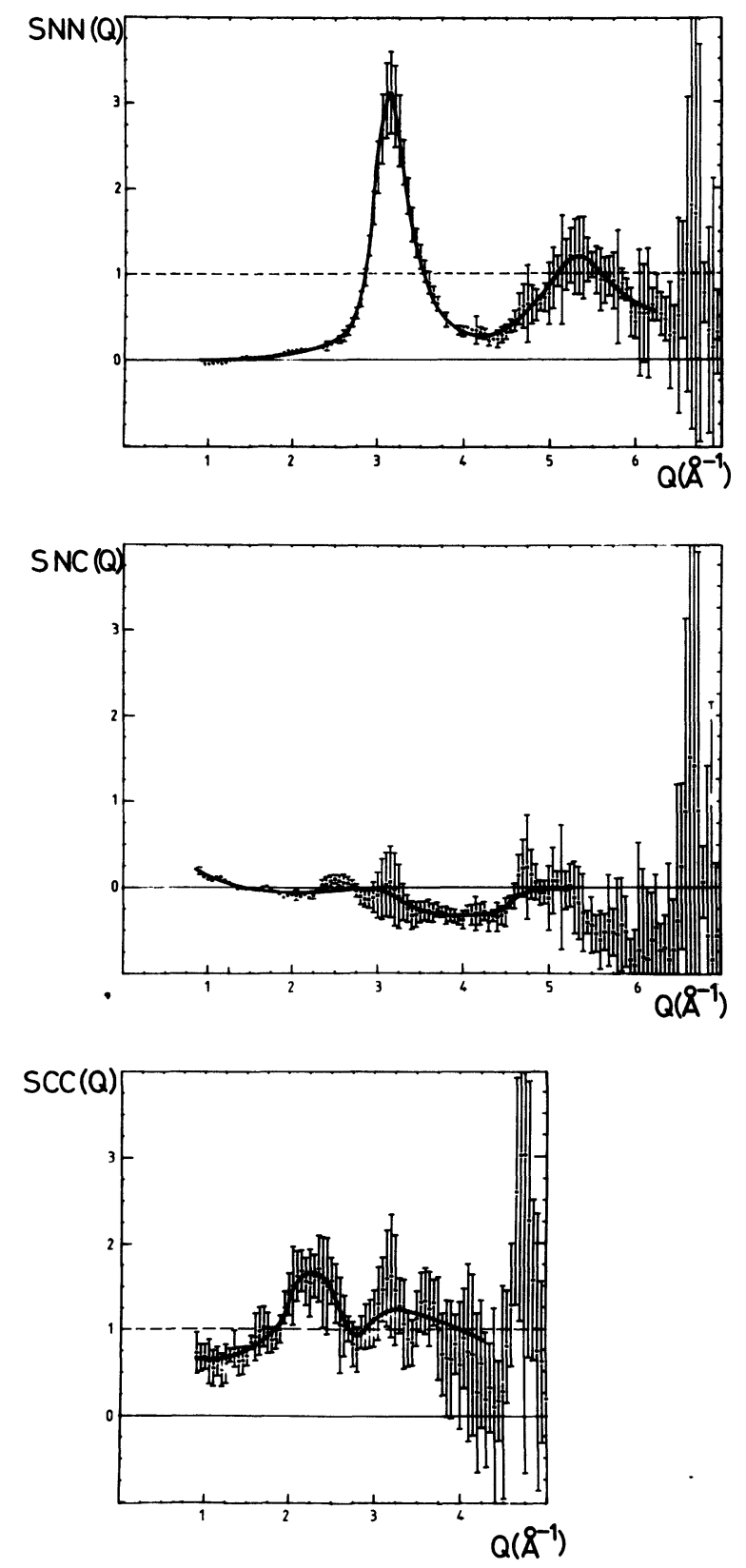

Fig. 5. - Facteurs de structure partiels Bhatia et Thornton des alliages Co-P amorphes.

[Bhatia-Tornton partial structure factors of an amorphous $\mathrm{Co}_{83} \mathrm{P}_{17}$ alloy.] prendrons comme exemple le cas des alliages ferromagnétiques cobalt-phosphore où les facteurs de structure partiels ont été déterminés par la méthode des neutrons polarisés avec une précision raisonnable [17], [18].

La figure 5 reproduit les facteurs de structure BT de l'alliage $\mathrm{Co}_{83} \mathrm{P}_{17}$. Elle montre que la fonction $S_{\mathrm{NC}}(Q)$ est quasiment nulle dans l'intervalle significatif : $1 \lesssim Q \lesssim 6 \AA^{-1}$. Il est donc possible d'utiliser l'approximation $S_{\mathrm{NC}}=0$ pour calculer les fonctions $S_{\mathrm{NN}}$ et $S_{\mathrm{CC}}$ à partir de deux diagrammes de diffraction. L'erreur systématique ainsi introduite est largement compensée par l'amélioration de la précision [18]. On tire alors les conclusions suivantes :

a) La fonction $S_{\mathrm{NN}}$ possède en

$$
Q_{1}=3,15 \pm 0,05 \AA^{-1}
$$

un premier maximum d'intensité : $S_{\mathrm{NN}}\left(Q_{1}\right)=3,1$. Son second maximum, situé en $Q_{2}^{\prime}=5,45 \AA^{-1}$, présente un épaulement en $Q_{2}^{\prime \prime}=6,15 \AA^{-1}$.

b) La fonction $S_{\mathrm{cc}}$ possède en $Q_{\mathrm{S}}=2,1 \pm 0,1 \AA^{-1}$ un prépic dont l'amplitude : $S_{\mathrm{cc}}\left(Q_{\mathrm{s}}\right)-S_{\mathrm{cc}}\left(Q_{\mathrm{s}} / 2\right)$ est voisine de 0,9 .

3.2 INTERPRÉTATION. - Examinons les résultats précédents à la lumière des modèles de sphères adhésives.

La quasi-nullité de la fonction $S_{\mathrm{NC}}$ montre que les atomes de cobalt et de phosphore ont des tailles voisines (ce qui exclut l'hypothèse interstitielle si souvent avancée). Il faut donc comparer les résultats expérimentaux aux modèles d'alliage de substitution.

L'existence d'un prépic dans la fonction $S_{\mathrm{CC}}$ indique un phénomène d'ordre chimique. La position de ce maximum de surstructure est en bon accord avec l'équation (23) et son amplitude permet d'évaluer le paramètre d'ordre des premiers voisins (Tableau I). On trouve : $\xi \simeq-0,22$. Cette valeur est pratiquement égale à la valeur minimum calculée à l'aide de la relation (24) : $\xi_{\min }=-0,21$ (pour $C_{\mathrm{p}}=0,17$ ). Les atomes de phosphore sont donc uniquement entourés d'atomes de cobalt conformément à l'hypothèse avancée par Sadoc et al. [39].

Enfin, la fonction $S_{\mathrm{NN}}$ devrait s'interpréter à l'aide des modèles d'amorphe monoatomique (\$ 2.4.2.2). En effet, les positions du premier et du deuxième maximum de $S_{\mathrm{NN}}$ avec son épaulement sont reproduites à $1 \%$ près si l'on admet que le paramètre de perfection des tétraèdres du réseau amorphe (global) est égal à : $K \simeq 1,05$ et le diamètre commun des atomes de cobalt et de phosphore à :

$$
d \simeq 2,44 \pm 0,04 \AA .
$$

Ce dernier résultat est plausible puisque les diamètres de Goldschmidt du cobalt et du phosphore valent respectivement : $d_{\mathrm{Co}}=2,50 \AA$ et $d_{\mathrm{P}}=2,18 \AA$. Toutefois, la compacité : $\gamma=0,68 \pm 0,04$ calculée à l'aide du diamètre $d$ et des mesures de densité de 
Cargill et Cochrane [47] est nettement supérieure à la valeur $\gamma=0,51$ déduite du modèle. De plus, l'intensité expérimentale $S_{\mathrm{NN}}\left(Q_{1}\right)=3,1 \mathrm{du}$ premier maximum est anormalement grande par rapport à son intensité théorique : $S_{\mathrm{NN}}\left(Q_{1}\right)=2,35(\S 2.3 .4)$.

Ces difficultés se retrouvent pour la plupart des amorphes métalliques et ne peuvent être surmontées à l'aide des modèles de sphères adhésives précédents. En effet, une augmentation du paramètre $K$ fait croître la compacité et l'intensité $S_{\mathrm{NN}}\left(Q_{1}\right)$ mais elle entraîne la disparition de l'épaulement sur le deuxième maximum de $S_{\mathrm{NN}}(\S 2.3 .4)$. D'autre part, l'introduction de lacunes ou de vibrations a des effets contraires au résultat souhaité ( $\S 2.3 .5$ et $\S 2.3 .6)$. Il faut donc avoir recours aux modèles plus élaborés présentés ci-après.

4. Derniers modèles. - 4.1 DES PRINCIPES CONTESTÉs. - Pour résoudre les difficultés précédentes, il faut remettre en question l'une des trois hypothèses de base des modèles de sphères adhésives présentés ci-dessus, à savoir :

- la non-directionnalité des liaisons interatomiques,

- la dureté infinie du potentiel de paire,

- ou l'additivité des diamètres atomiques.

Nous examinerons successivement ces trois tentatives.

4.2 Modèles DiReCtionnels. - En présence de liaisons covalentes à caractère directionnel la géométrie de l'ordre local se précise. C'est pourquoi Machizaud et Flechon [48], [49] puis Gaskell [50] ont proposé de décrire la structure des alliages amorphes métal de transition-métalloïde (B, C, P, Si) par des empilements désordonnés de prismes trigonaux analogues aux motifs observés dans les composés cristallins (borures, carbures, phosphures, siliciures). Comme ces motifs sont formés d'un métalloïde entouré de 9 atomes métalliques, le modèle reproduit bien l'ordre chimique maximum observé dans les alliages Co-P. De plus l'intensité théorique du premier maximum de $S_{\mathrm{NN}}$ soit $: S_{\mathrm{NN}}\left(Q_{1}\right) \simeq 2,7$ se rapproche de la valeur 3,1 expérimentale. Un progrès notable semble ainsi obtenu.

D'autre part, dans son étude des agrégats formés lors de la détente d'un jet libre d'argon, J. Farges [51] a prouvé que les effets de surface peuvent stabiliser certains polyèdres réguliers. En effet, cet auteur a montré que les agrégats d'argon contenant moins de 700 atomes ont une structure icosaédrique (qui se manifeste par de petits détails sur les diagrammes de diffraction électronique) et que ces agrégats évoluent vers une structure cubique à faces centrées au-delà de 700 atomes.

Par ailleurs, la structure icosaédrique a souvent été identifiée à l'ordre local dans les amorphes car sa symétrie 5 empêche toute périodicité [35]. Les observations de Farges indiquent que cette hypothèse est plausible pour des grains amorphes très fins. Il serait donc intéressant de comparer la structure des amorphes massifs à des modèles nouveaux comportant des noyaux icosaédriques raccordés entre eux par des régions plus désordonnées.

4.3 ModÈles RELAXÉS. - Yamamoto et al. [52] font relaxer un réseau compact amorphe de sphères adhésives $(\$ 2.3 .2)$ en utilisant un potentiel interatomique mou. La suppression de la contrainte d'impénétrabilité des sphères permet à ces auteurs d'atteindre une compacité : $\gamma=0,68$. Simultanément l'intensité du premier pic de $S(Q)$ augmęnte jusqu'à 3,3 et un épaulement apparaît sur le deuxième pic de $S(Q)$ ! Cette méthode de relaxation est donc très fructueuse puisqu'elle résout tous les problèmes en suspens ( $\$ 3.2$ ). Toutefois la précision (statistique) atteinte par ces modèles est encore limitée car ils ne comprennent pas plus de 1000 particules [18].

4. 4 MODÈLES DE SPHÈRES DURES NON ADDITIVES. Les mélanges de sphères dures à diamètres non additifs ont été étudiés dans le cadre de la théorie de Percus-Yevick [22], [53], de la théorie des cavités [54], [55], [56], [57] ou des calculs de Monte-Carlo [58]. Selon que la distance $d_{12}$ est inférieure ou supérieure à la demi-somme des distances $d_{11}$ et $d_{22}$, ces modèles pourraient décrire les propriétés thermodynamiques des sels fondus [56] ou des alliages liquides à démixtion [55], [57]. Malheureusement, il n'a pas été possible jusqu'ici de calculer analytiquement les facteurs de structure partiels correspondant à ces potentiels [53].

5. Conclusion. - Au terme de cette revue, il apparaît souhaitable d'orienter les expériences de diffraction par les alliages amorphes vers la détermination des facteurs de structure partiels de Bhatia et Thornton [3]. La caractérisation de la structure géométrique exige des mesures très précises d'intensité permettant d'observer la hauteur $S_{\mathrm{NN}}\left(Q_{1}\right)$ du premier pic de la fonction $S_{\mathrm{NN}}$, l'épaulement de son deuxième pic et éventuellement les bosses caractéristiques d'un ordre de type polyédrique [49], [51].

Pour déterminer l'ordre chimique, il faut d'autre part concentrer son attention sur l'intervalle de $Q$ : $0 \lesssim Q \lesssim Q_{1}$ car c'est là qu'apparaissent les prépics ou les préminimums caractéristiques des phénomènes d'ordre ou de ségrégation dans la fonction $S_{\mathrm{CC}}$ [5], [17]. Les effets de taille se manifestent enfin par les oscillations du facteur de structure $S_{\mathrm{NC}}$ [5].

Il est donc possible d'accéder à l'ordre local moyen à l'aide des expériences de diffraction. Toutefois, seules les techniques de diffusion centrale [59] ou de champ hyperfin (résonance magnétique nucléaire, effet Mössbauer, diffusion inélastique des neutrons avec spin flip [60]) permettent d'étudier les fluctuations par rapport à cet environnement moyen.

D'un point de vue théorique, les modèles géo- 
métriques de sphères adhésives [5] rendent compte de l'ordre local observé dans les amorphes métalliques de façon satisfaisante. Les derniers raffinements de ces modèles prenant en compte les effets directionnels (covalents) [49], [50], la mollesse des potentiels de paire [52] ou la non-additivité des diamètres ato- miques [53] devraient conduire à un accord parfait avec les résultats expérimentaux.

Ces progrès sont très encourageants. Cependant le travail à accomplir reste considérable car les verres métalliques représentent un lopin dans le vaste domaine des amorphes.

\section{Bibliographie}

[1] Fournet, G., C.R. Hebd. Séan. Acad. Sci. 229 (1949) 1071,

[2] Faber, T. E., Ziman, J. M., Phil. Mag. 11 (1965) 153.

[3] Bhatia, A. B., Thornton, D. E., Phys. Rev. B 2 (1970) 3004.

[4] Edwards, F. G., Enderby, J. E., Howe, R. A., Page, D. I., J. Phys. C 8 (1975) 3483.

[5] BLETRY, J., Z. Naturforsch. a 33 (1978) 327.

[6] WineYARD, G. H., Liquid metals and solidification (Am. Soc. for Metals, Cleveland Ohio), 1958.

[7] Ramesh, T. G., Ramaseshan, S., J. Phys. C 4 (1971) 3029.

[8] Waseda, Y., Tamaki, S., Phil. Mag. 32 (1975) 951.

[9] Waseda, Y., Tamaki, S., Z. Phys. B 23 (1976) 315.

[10] Waseda, Y., Tamaki, S., Comm. Phys. 1 (1976) 3.

[11] EGGER, H., Communication privée après essai.

[12] Enderby, J. E., North, D. M., Egelstaff, P. A., Phil. Mag. 14 (1966) 961.

[13] Page, D. I., Mika, K., J. Phys. C 4 (1971) 3034.

[14] Derrien, J. Y., DupuY, J., J. Physique 36 (1975) 191.

[15] Mitchell, E. W. J., Poncet, P. F. J., Stewart, R. J., Phil. Mag. 34 (1976) 721.

[16] Bellissent Funel, M. C., Desre, P. J., Bellissent, R., Tourand, G., J. Phys. F 7 (1977) 2485.

[17] Bletry, J., Sadoc, J. F., J. Phys. F 5 (1975) L 110.

[18] Bletry, J., Thèse d'Etat, Grenoble (1979).

[19] Ruppersberg, H., Egger, H., J. Chem. Phys. 63 (1975) 4095.

[20] Reiter, H., Ruppersberg, H., Speicher, W., Proc. 3rd Int. Conf. on Liquid Metals 133 (Bristol 1976).

[21] RUPPERSBERG, H., KNOLL, W., Z. Naturforsch. a 32 (1977) 1374.

[22] Percus, J. K., Yevick, G. J., Phys. Rev. 110 (1958) 1.

[23] Thiele, E., J. Chem. Phys. 39 (1963) 474.

[24] Wertheim, M. S., Phys. Rev. Lett. 10 (1963) 321.

[25] Baxter, R. J., J. Chem. Phys. 49 (1968) 2770.

[26] Alder, B. J., Wainwright, T. E., J. Chem. Phys. 31 (1959) 459.

[27] Rahman, A., Phys. Rev. 136 (1964) A 405.

[28] Hansen, J. P., Schiff, D., Mol. Phys. 25 (1973) 1281.

[29] Lantelme, F., TurQ, P., Quentrec, B., Lewis, J. W. E., Mol. Phys. 28 (1974) 1537.

[30] Mathieu, J. C., Thèse d'Etat, Grenoble (1967).

[31] Mathieu, J. C., Durand, F., Bonnier, E., J. Chim. Phys. 62 (1965) 88.

[32] Laty, P., Joud, J. C., Mathieu, J. C., Desre, P., Phil. Mag. B 38 (1978) 1.
[33] Hicter, P., Thèse d'Etat Grenoble (1969).

[34] Hicter, P., Durand, F., Bonnier, E., J. Chim. Phys. 68 (1971) 804

[35] Dixmier, J., Thèse d'Etat, Orsay (1969).

[36] Polk, D. E., J. Non Cryst. Sol. 5 (1971) 365.

[37] Bernal, J. D., Proc. Roy. Soc. London A 280 (1964) 299.

[38] Finney, J. L., Proc. Roy. Soc. London A 319 (1970) 479.

[39] Sadoc, J. F., Dixmier, J., Guinier, A., J. Non Cryst. Sol. 12 (1973) 46

[40] Ichikawa, T., Phys. Status Solidi a 29 (1975) 293.

[41] BleTry, J., Z. Naturforsch. 32 a (1977) 445.

[42] Ornstein, L. S., Zernike, F., Proc. Acad. Sci. Amsterdam 17 (1914) 793.

[43] Lemaignan, C., Communication privée.

[44] Schütte, K., VAN DeR W Aerden, B. L., Math. Annalen 123 (1951) 96.

[45] O'Leary, W. P., J. Phys. F 5 (1975) L 175.

[46] Kudo, T., Mizoguchi, T., Watanabe, N., Nimura, N., Mizawa, M., Suzuki, K., J. Phys. Soc. Jap 45 (1978) 1773.

[47] Cargill, G. S., III, Cochrane, R. W., J. Physique Colloq. 35 (1974) C4-269.

[48] Machizaud, F., Thèse d'Etat, Nancy (1973).

[49] Flechon, J., Machizaud, F., Marchal, G., Revue Phys. Appl. 7 (1972) 169.

[50] Gaskell, P. H., J. Non Cryst. Sol 32 (1979) 207.

[51] FARGES, J., Thèse d'Etat, Orsay (1977).

[52] Yаmamoto, R., Matsuoka, H., Doyama, M., Phys. Lett. 64A (1978) 457.

[53] Lebowitz, J. L., Zomick, D., J. Chem. Phys. 54 (1971) 3335.

[54] Reiss, H., Frisch, H. L., Lebowitz, J. L., J. Chem. Phys. 31 (1959) 369.

[55] Ruelle, D., Phys. Rev. Lett. 27 (1972) 1039.

[56] Bergmann, E., Tenne, R., Chem. Phys. Lett. 56 (1978) 310.

[57] Tenne, R., Bergmann, E., Phys. Rev. A 17 (1978) 2036.

[58] Adams, D. J., Mac Donald, I. R., J. Chem. Phys. 63 (1975) 1900.

[59] GUINIER, A., Théorie et technique de la radiocristallographie (Dunod) 1964.

[60] Heidemann, A., Z. Phys. 238 (1970) 208. 